\title{
Clubbing, Arthralgia and a Large Intrathoracic Mass
}

\author{
Marjorie Lorillou ${ }^{a}$ Françoise Thivolet-Béjui ${ }^{\mathrm{b}}$ François $\operatorname{Tronc}^{\mathrm{c}}$ Nicolas Girard ${ }^{\mathrm{a}}$ \\ Jean-François Cordier ${ }^{\mathrm{a}}$ Vincent Cottin ${ }^{\mathrm{a}}$ \\ a Service de pneumologie - Centre de référence national des maladies pulmonaires rares et Centre de \\ compétences de I'hypertension artérielle pulmonaire, ${ }^{b}$ Centre de Pathologie Est, and ${ }^{\mathrm{c} S e r v i c e}$ de chirurgie \\ thoracique, Hôpital Louis Pradel, Hospices Civils de Lyon, Université Lyon I, Lyon, France
}

\section{Case Report}

A 65-year-old man was referred to our department in 2008 for mild dyspnea. The patient was a never-smoker, and had no history of asbestos exposure. In 2006, he had presented an episode of fever, polyarthralgia and erythema of the fingers that resolved spontaneously; at that time the chest radiograph had demonstrated a dense opacity of the left base that was considered an infectious pneumonia (fig. 1), with clinical improvement with antibiotic therapy and he was lost to follow-up. However, he subsequently presented with chronic arthralgia, mild fever, night-sweats and fatigue, which worsened progressively between 2006 and 2008. A rheumatologist considered the diagnosis of rheumatoid arthritis.

Upon referral to our center, clinical examination demonstrated erythematous clubbing of the extremities of the fingers (fig. 2) and toes, and dullness of the left hemithorax. The chest radiograph showed a left basal opacity (fig. 3) that had increased in size when compared to 2006. A chest CT scan further showed a mass measuring $15 \times$ $11 \mathrm{~cm}$ in diameter, located in the basal area of the left

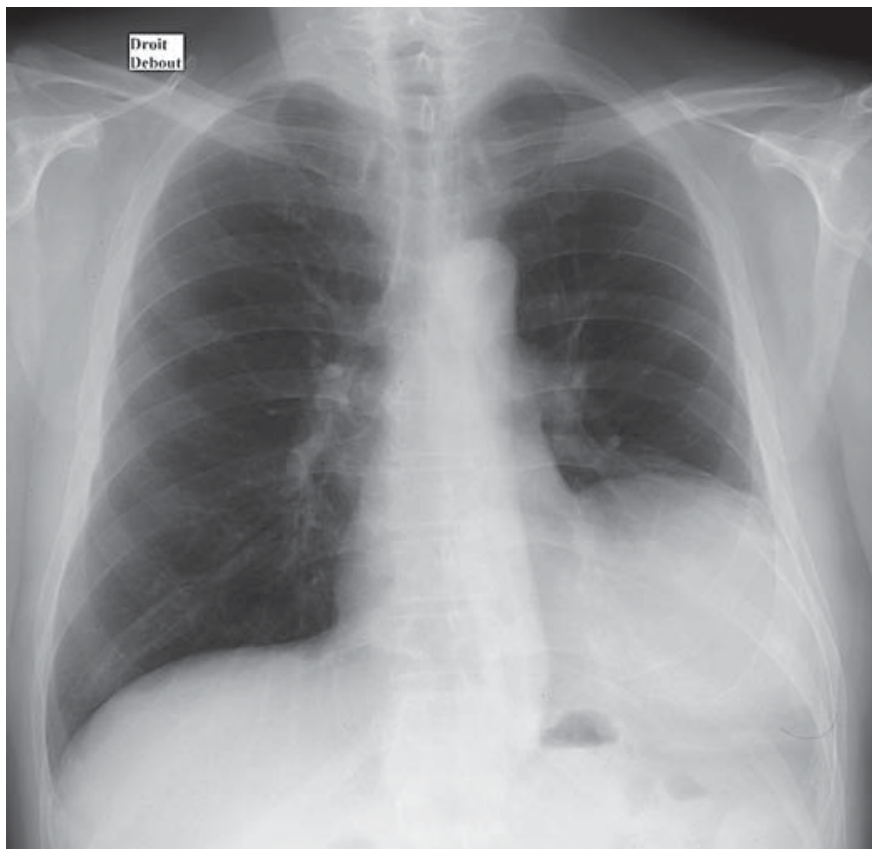

Fig. 1. Chest radiograph in 2006 showing a mass of the left hemithorax.

\section{KARGER}

Fax +4161306 1234

E-Mail karger@karger.ch

www.karger.com
(C) 2012 S. Karger AG, Basel

0025-7931/12/0842-0150\$38.00/0

Accessible online at:

www.karger.com/res
Vincent Cottin

Hôpital Louis Pradel

FR-69677 Lyon Cedex (France)

Tel. +33 472357072

E-Mail vincent.cottin@chu-lyon.fr 


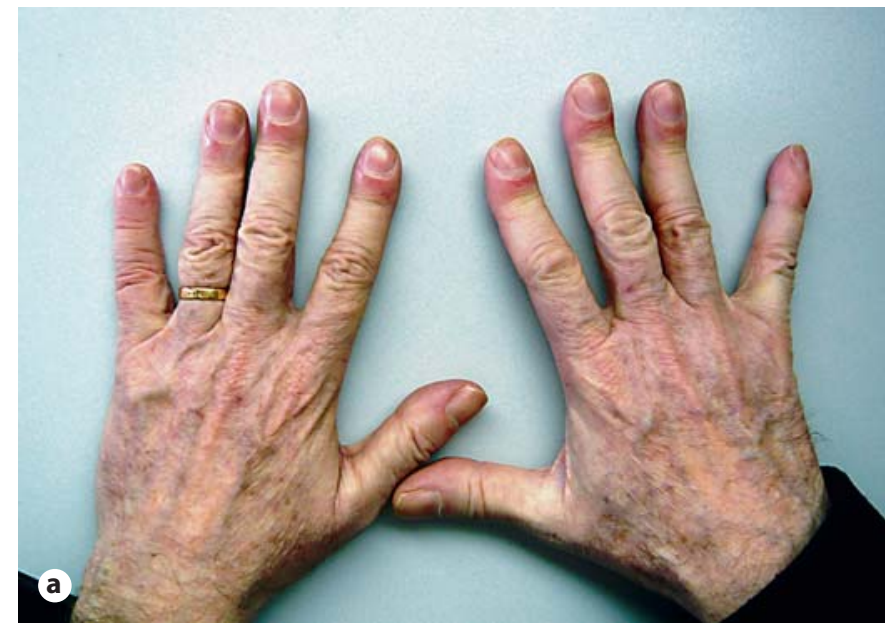

Fig. 2. Finger clubbing at presentation. a Macroscopic appearance. $\mathbf{b}$ Tc $99 \mathrm{~m}$ bone scintigraphy at presentation.
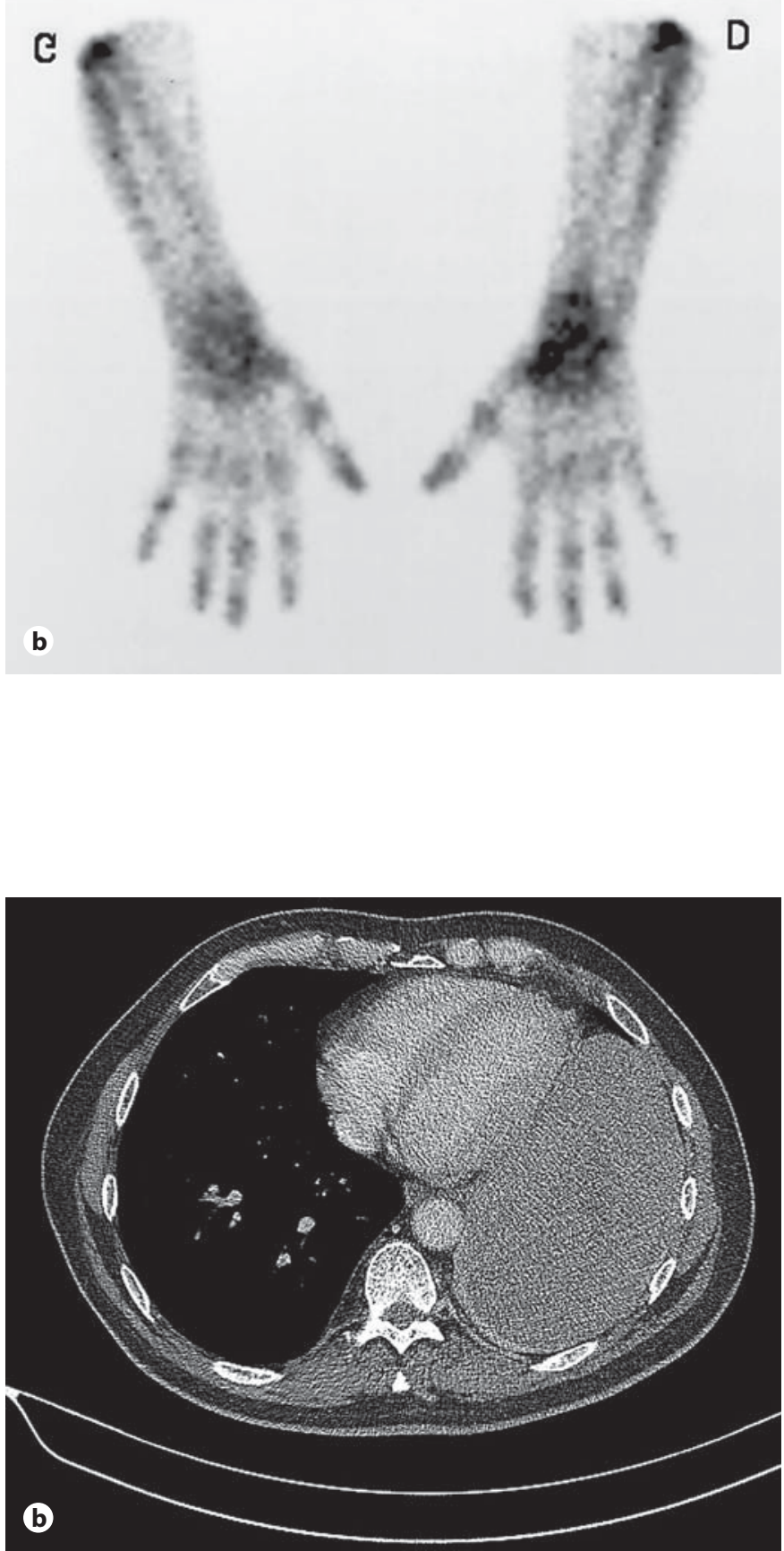

Fig. 3. Chest imaging at presentation in 2008. a Radiograph showing that the mass of the lower left hemithorax had increased in size. b Chest CT scan showing a large, homogeneous solid mass in the lower left hemithorax. No pleural abnormality of chest wall invasion is seen.

hemithorax. No mediastinal lymphadenopathy was seen. With contrast enhancement, the mass was heterogeneous in density and, regular in shape, with no air bronchogram and no invasion of adjacent structures. Fiberoptic bronchoscopy showed extrinsic stenosis of the left lower lobar bronchus. Bronchial biopsies were unremarkable. A bone scintigraphy showed a typical pattern of periostic hyperfixation, suggesting paraneoplastic hypertrophic pulmonary osteoarthropathy (fig. 2).

What is your diagnosis? 


\section{Diagnosis: Solitary Fibrous Tumor of the Pleura}

Further preoperative work-up with 18 -flurodeoxyglucose (FDG) positron emission tomography showed a moderate FDG uptake of the lung mass (SUV max 2.9), with an absence of extrathoracic hyperfixation. A benign tumor was suspected. The patient underwent a left posterior lateral thoracotomy, demonstrating a large, well-circumscribed tumor, with necrosis involution and attached to the pleura by a pedicle. The tumor did not invade the lung parenchyma, pleura or chest wall. Wedge resection was performed and the tumor measured $17 \times 16 \times 11 \mathrm{~cm}$ $(1,400$ g). Histological analysis (fig. 4) identified a benign solitary fibrous tumor of the pleura (SFTP) with a pedunculated insertion on visceral pleura and on the lingula, suggesting pleural origin. The tumor stained positive (fig. 4) for vimentin, CD34, Bcl-2 and CD99, and stained negative for cytokeratin KL1 and S100. Neither extra-tumor invasion nor lymphadenopathy was present. Ki-67 staining was observed in less than $2 \%$ of cells, leading to the diagnosis of a low-grade, so-called 'benign' SFTP.

Upon resection of the tumor, the patient experienced a favorable outcome with the immediate disappearance of systemic symptoms, especially arthralgia and dyspnea. Clubbing of the fingers and toes resolved within 6 months. Four years after surgery, the patient is free of symptoms.

Fibrous tumors are rare tumors deriving from mesenchymal cells, benign in $80-90 \%$ of cases $[1,2]$. They have been reported in a number of anatomic locations, with SFTP being the most common [1]. SFTP predominates in the sixth or seventh decade [1], with no link to genetic factors or tobacco or asbestos exposure [2]. Half of the patients are asymptomatic at diagnosis, with possible chest pain or dyspnea when the tumor progresses to up to $37 \mathrm{~cm}$ in diameter (thereby possibly compressing adjacent structures) [2]. Although SFTP are usually benign tumors, hypertrophic pulmonary osteoarthropathy and clubbing are reported in up to $20 \%$ of cases $[2,3]$, and classically resolve shortly after tumor resection, suggesting they are related to putative circulating mediators secreted by tumor cells [4]. Tumor cells may produce hyaluronic acid, resulting in periosteal bone changes with chronic hypoxia and secretion of vascular endothelial growth factor and platelet-derived growth factor $[5,6]$. More rarely (4\%), SFTP may present with intractable paraneoplastic hypoglycemia [2], especially in women and in tumors located in the right hemithorax [1] and resulting from the secretion of incompletely processed insulin-like growth factor type-2 [7-9].
This case was typical of benign SFTP at imaging, with a homogeneous, well-demarcated tumor pedunculated to the visceral pleura (in half the cases), with the same density as muscle and possible contrast enhancement on CT scan. Calcification may be observed in a third of cases. There is no or minimal FDG uptake on positron emission tomography. The differential diagnosis includes other pleural-based tumors, in particular mesothelioma [10] and sarcoma. The diagnosis is based on histology obtained by transthoracic biopsy [6] or, preferably, surgical resection. Immunostaining for $\mathrm{CD} 34$ and the absence of staining for cytokeratin differentiates SFTP from other pleural-based tumors, especially mesothelioma [1]. Relapse after curative resection has been reported in less than $10 \%$ of cases, corresponding to high-grade proliferation referred to as 'malignant' SFTP [1], with extensive infiltration of the pleura and often precluding complete resection to be achieved.

\section{Key Words}

Fibrous solitary tumor - Pleura • Mesenchymal cell • Orphan thoracic oncology
Lorillou/Thivolet-Béjui/Tronc/Girard/ Cordier/Cottin 

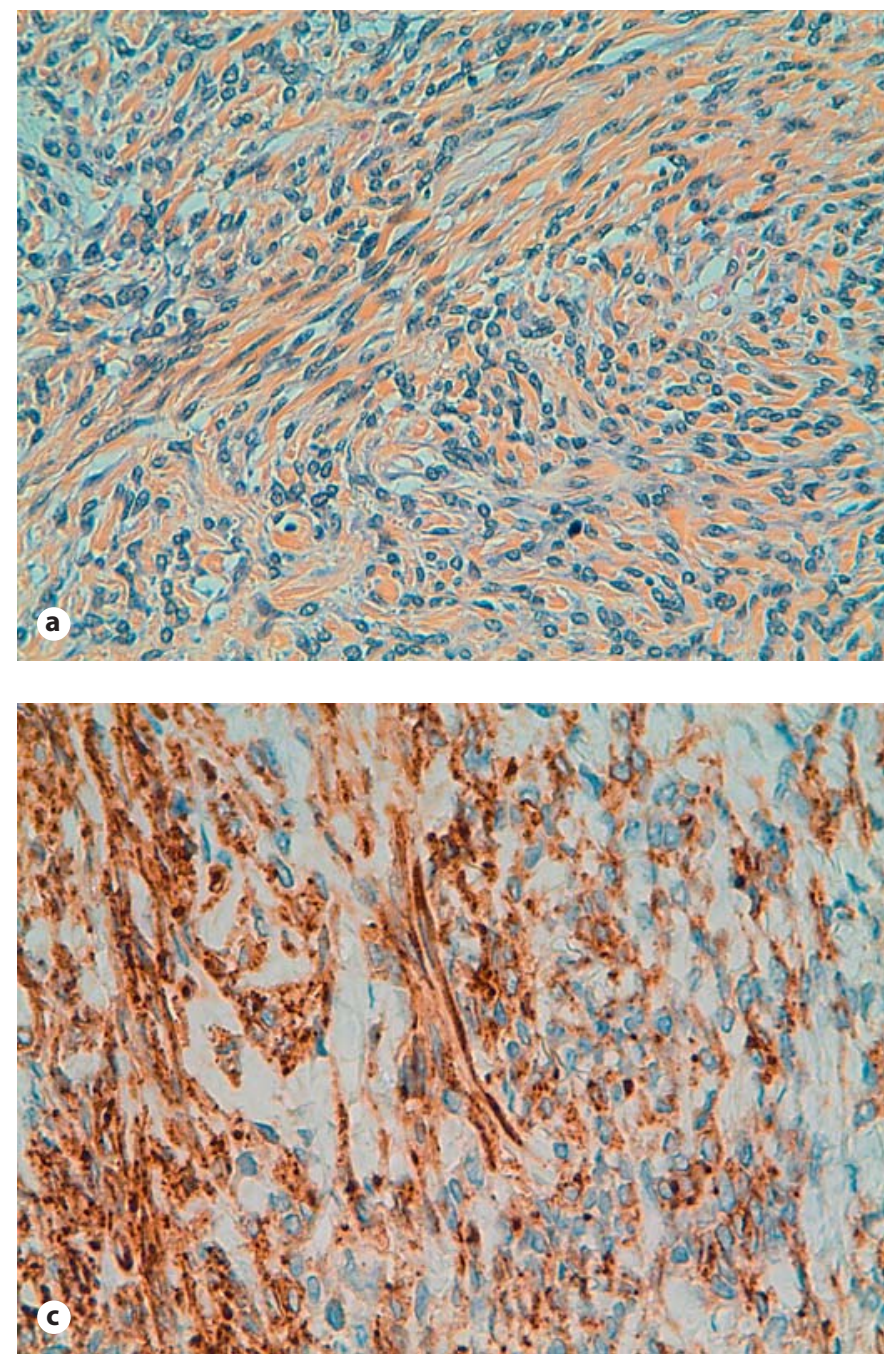

Fig. 4. Microscopic examination of the tumor. $\times 40$. a Staining with hematoxylin-eosin-saffron showing multiple interlacing fascicles of spindle cells and collagen fibers. b-e Immunostaining using vimentin (b), CD34 (c), bcl2 (d) and Ki-67 (e) antibodies.
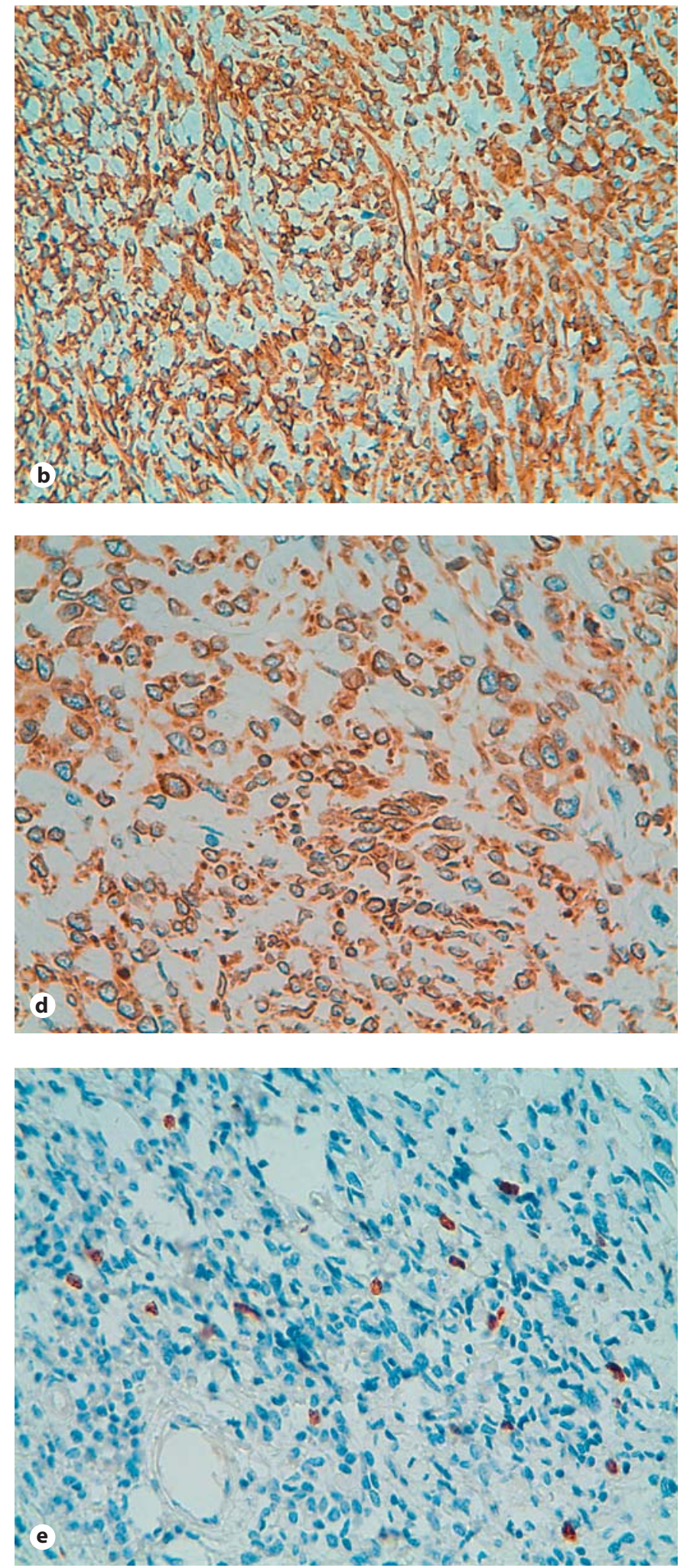


\section{References}

$>1$ England DM, Hochholzer L, McCarthy MJ: Localized benign and malignant fibrous tumors of the pleura. A clinicopathologic review of 223 cases. Am J Surg Pathol 1989; 13: 640-658.

$>2$ Briselli M, Mark EJ, Dickersin GR: Solitary fibrous tumors of the pleura. Eight new cases and review of 360 cases in the literature. Cancer 1981;47:2678-2689.

3 Hiraoka K, Morikawa T, Ohbuchi T, Katoh $\mathrm{H}$ : Solitary fibrous tumors of the pleura: clinicopathological and immunohistochemical examination. Interact Cardiovasc Thorac Surg 2003;2:61-64.
4 Atkinson S, Fox SB: Vascular endothelial growth factor (VEGF)-A and platelet-derived growth factor (PDGF) play a central role in the pathogenesis of digital clubbing. J Pathol 2004;203:721-728.

5 Kalebi AY, Hale MJ, Wong ML, Hoffman T, Murray J: Surgically cured hypoglycemia secondary to pleural solitary fibrous tumour: case report and update review on the Doege-Potter syndrome. J Cardiothorac Surg 2009;4:45.

6 Sun ZG, Wang Z, Zhang M: A 70-year-old man with hypoglycemia, clubbing of fingers and toes, and a large mass of the right hemithorax. Chest 2011;139:1528-1531.

$>7$ Tsuro K, Kojima H, Okamoto S, Yoshiji H, Fujimoto M, Uemura M, et al: Glucocorticoid therapy ameliorated hypoglycemia in insulin-like growth factor-II-producing solitary fibrous tumor. Intern Med 2006;45: 525-529.
Le Roith D: Tumor-induced hypoglycemia. N Engl J Med 1999;341:757-758.

$\checkmark 9$ Daughaday WH: Hypoglycemia due to paraneoplastic secretion of insulin-like growth factor-I. J Clin Endocrinol Metab 2007;92: 1616.

10 Tanrikulu AC, Abakay A, Kaplan MA, Kucukoner M, Palanci Y, Evliyaoglu O, et al: A clinical, radiographic and laboratory evaluation of prognostic factors in 363 patients with malignant pleural mesothelioma. Respiration 2010;80:480-487. 